\title{
Antiatherogenic Effect of Simvastatin Is Not Due to Decrease of LDL Cholesterol in Ovariectomized Golden Syrian Hamster
}

\author{
J. PITHA ${ }^{1}$, D. BOBKOVÁ ${ }^{1}$, J. KOVÁŘ ${ }^{1}$, J. HAVLÍČKOVÁ $^{2}$, R. POLEDNE ${ }^{1}$ \\ ${ }^{1}$ Laboratory for Atherosclerosis Research, Institute for Clinical and Experimental Medicine, Prague, \\ Czech Republic, ${ }^{2}$ Department of Experimental Medicine, Institute for Clinical and Experimental \\ Medicine, Prague, Czech Republic
}

Received March 10, 2009

Accepted June 24, 2009

On-line August 12, 2009

\section{Summary}

The changes of the composition of blood lipoproteins caused by menopause could also change the effect of hypolipidemic therapy. Using an experimental model we studied the changes of serum lipids and the effect of immediate or delayed treatment with simvastatin on atherosclerosis after surgical menopause. Female golden Syrian hamster aged 6 months were fed hypercholesterolemic diet during the whole study. Atherosclerotic changes in thoracic and abdominal aortas were assessed by stereomicroscopic method after 12 weeks. Four experimental groups were studied: sham-operated animals $(n=5)$, ovariectomized animals $(n=9)$, ovariectomized animals treated for 12 weeks $(n=10)$, and ovariectomized animals treated 4 weeks after ovariectomy for 8 weeks $(n=9)$. The dose of simvastatin was $10 \mathrm{mg} / \mathrm{kg}$ of body weight. After 12 weeks, ovariectomized animals had tenfold higher concentration of triglycerides in LDL fraction and significantly higher prevalence of atherosclerosis than animals without ovariectomy. Treatment with simvastatin substantially decreased the prevalence of atherosclerotic changes, but otherwise did not change individual serum lipids including LDL cholesterol. However, it improved proportions of pro- and antiatherogenic serum lipids mainly by the increase of HDL cholesterol. The timing of simvastatin treatment had no significant effect on atherosclerotic changes or lipid parameters. Simvastatin treatment partly prevented atherosclerotic changes induced by ovariectomy. This effect was not mediated by decrease of LDL cholesterol, but by increase in HDL cholesterol.

\section{Key words}

Ovariectomy - LDL particles - Triglycerides - Simvastatin • Atherosclerosis $\bullet$ Hamster

\section{Corresponding author}

Jan Pitha, Laboratory for Atherosclerosis Research, Institute for Clinical and Experimental Medicine, Videnska 1952/8, 14021 Praha (Prague) 4, Czech Republic. Fax: +0420 241271574. E-mail: japi@ikem.cz

\section{Introduction}

More women than men are affected by clinical manifestation of atherosclerosis in absolute numbers due to increased life expectancy. Furthermore, in spite of that women before menopause are generally at low risk of cardiovascular disease, after they develop coronary heart disease they have a worse prognosis than men (Milcent et al. 2007). Prevention measures, based on the knowledge of underlying pathophysiological mechanisms, are therefore of critical importance in this group, but those currently used are based on data obtained mainly in men.

The loss of protection by female hormones after menopause is still considered among the most important causes of atherosclerosis progression. However, hormone replacement therapy has not been of benefit in clinical trials (Raza et al. 2004), even though there is considerable epidemiological and basic science evidence that indicates the contrary. The route (Vráblík et al. 2008) and timing (Clarkson 2007) of hormone administration could be of importance to achieve better clinical results in hormone replacement therapy in women.

Hypolipidemic therapy mainly with 3-hydroxy3-methyl-glutaryl-CoA reductase inhibitors (statins) significantly lowered cardiovascular mortality and 
morbidity. However, the data obtained in women are based mainly from subanalyses done in studies focused mainly on the men population in spite of potential gender related difference in circulating lipoproteins and their modification by environmental inputs (Frohlich et al. 2004, Kovár and Poledne 2008). Therefore, the hypolipidemic treatment originally tailored for men could differently affect lipoproteins levels in women and could be dependent on their reproductive/hormonal status (Walsh and Pignone 2004). If we were able to define the effect of hypolipidemic drugs in women more precisely, their treatment could be even more efficient.

In recent experimental studies in rat, estrogen deprivation resulted in the vessel wall becoming more vulnerable to atherogenic stimuli (Arenas et al. 2006). It was caused by up-regulation of tumor necrosis factor-alfa enhancing adrenergic vascular constriction and altering the vascular angiotensin II system. These changes occur very soon after surgical menopause. Therefore, potential changes in the composition of atherogenic serum lipoproteins in menopause could accelerate the progression of atherosclerosis in this period and attenuate treatment effect of hypolipidemic therapy. Changes in lipoprotein composition in menopause and their impact on the antiatherogenic effect of hypolipemic therapy are yet to be studied. Therefore, the timing of hypolipemic therapy with regard to reproductive stages could also be of importance.

The aim of our study was to assess the effect of menopause on the composition of serum lipoproteins and atherosclerotic changes and their impact on the effect of immediate or delayed statin treatment in the model of golden Syrian hamster.

\section{Methods}

We have chosen this particular experimental model because it has been proved to be very good model for studying lipid metabolism together with atherosclerotic changes, which are quite similar to those in humans and are detectable already after 4 weeks of atherogenic diet (Lucas et al. 2001, Nistor et al. 1987, Sohn et al. 1999, Wilson et al. 1999).

Studies were performed in accordance with the guidelines and practices established by the Institute for Clinical and Experimental Medicine Animal Care and Use Committee and are in the accordance with laws in the Czech Republic. All animals in the present study were housed in facilities accredited by the Czech Association for Accreditation of Laboratory Animal Care. This study was designed to be performed on females at the age of 6 months.

Four experimental groups were studied in 12-week period as follows: animals undergoing sham operation $(\mathrm{n}=5)$, ovariectomized animals without any therapy $(n=9)$, animals treated with simvastatin immediately after ovariectomy for 12 weeks $(\mathrm{n}=10)$, and animals treated with simvastatin 4 weeks after ovariectomy for 8 weeks ( $\mathrm{n}=9$ ).

All surgical procedures were done in general anesthesia with ketamine and flunitrazepam. The dosage was adjusted to fully anesthetize the animals to avoid any painful reactions during and after the surgery. During the whole study period (before and after ovariectomy), the animals were fed hypercholesterolemic non-purified diet containing $10 \%$ coconut oil and $0.05 \%$ cholesterol, which is expected to simulate effects of high-cholesterol diet in humans according to already published data (Wilson et al. 1999). Otherwise, they had free access to food and liquids. The dose of statins was $10 \mathrm{mg}$ of simvastatin (Zocor, MSD, USA) $/ \mathrm{kg}$ of body weight added to chow diet as was already published (El-Swefy et al. 2000).

At the time of sacrifice, 12 weeks after ovariectomy, animals were anesthetized with intraperitoneal injection of sodium pentobarbital ( $50 \mathrm{mg} / \mathrm{kg}$ body weight). Their chest was opened and blood was collected in a heparin tube from the puncture to the right ventricle. Ascending aorta was canulated and thoracic and abdominal aorta was repeatedly flushed with isotonic (physiological) saline solution. The thoracic and abdominal aorta were completely dissected of all surrounding tissue and stretched and pinned down, moistured by saline. Immediately after removal, pictures of thoracic and abdominal aortas were recorded on camera (digital camera Olympus - Camedia, Zoom 3030, Japan). Presence of atherosclerotic changes was assessed by stereomicroscopic method (magnified 8 and 16 times). Obtained images were assessed by two observers (JP and DB.). If both observers were in accordance, aortas were labeled as area with atherosclerotic plaques. In the case of disagreement the aortas were labeled as without plaques (one case in ovariectomized group with statin treatment for 8 weeks). Aortas with and without atherosclerotic changes are shown in Figure 1.

\section{Serum lipids}

After sacrifice, the serum was immediately 
a

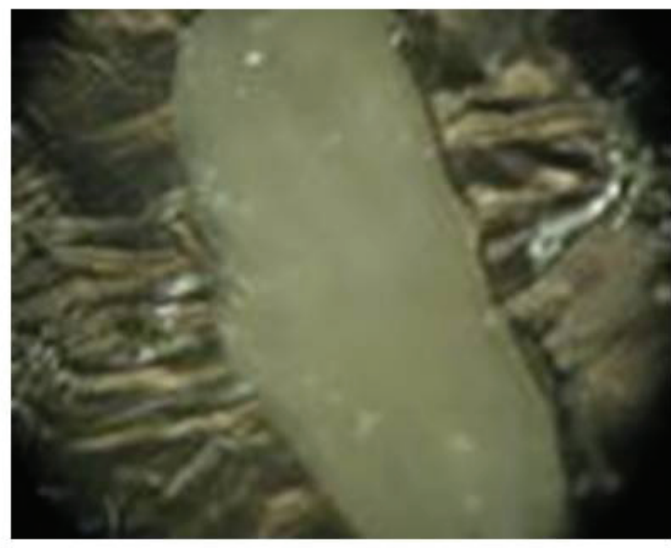

b

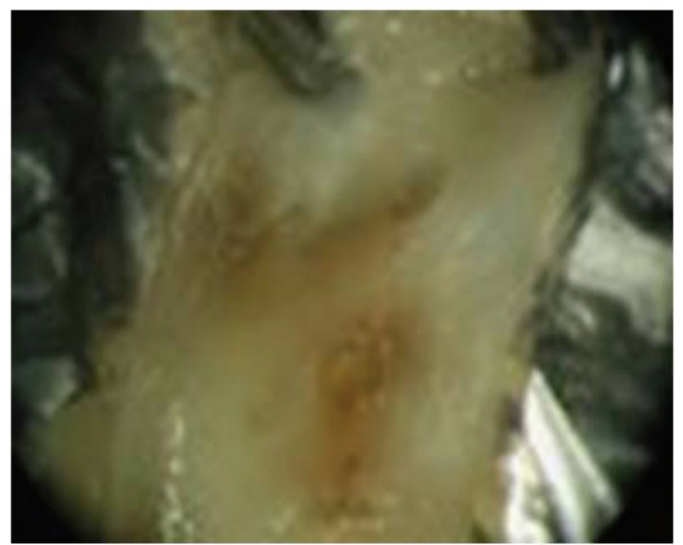

Fig. 1. Abdominal aorta without (a) and with (b) atherosclerotic changes in golden Syrian hamster. Stereomicroscopic method (enlarged 16 times).

separated for lipid measurement. Whole blood samples were centrifuged at 5,000 $\mathrm{g}$ for $5 \mathrm{~min}$ at $4{ }^{\circ} \mathrm{C}$ and serum was collected. Serum cholesterol and triglycerides were measured in each animal by fully automated (Cobas Mira $\mathrm{S}$ auto analyzer) enzymatic method (reagents from Hoffmann-LaRoche, Basel, Switzerland). Cholesterol and triglycerides content in HDL, LDL, IDL and VLDL fractions were measured in pooled serum for each group after sequential ultracentrifugation (Havel et al. 1955) by RIA method (Stratec, RIA Immunotech, USA). Atherogenic index was calculated as total cholesterol divided by HDL cholesterol. In addition, atherogenic index of plasma (AIP) related to the particle size of lipoproteins was calculated as log (triglycerides/HDLcholesterol) (Dobiášová and Frohlich 2001).

\section{Statistical analyses}

Data are presented as the absolute and relative frequencies for categorical variables and means with standard deviations for continuous ones. Differences of means were tested by ANOVA. The Fischer exact test was used for comparison of proportions. In the case of pooled samples we did not use formal statistical tests and we only described observed differences.

\section{Results}

No significant differences in body weight between studied groups were found before and at the end of the experiment. Medians of body weight were $111 \mathrm{~g}$ at the beginning and $110 \mathrm{~g}$ at the end of experiment. Serum estradiol at the end of the study was lower in ovariectomized animals than in controls (ovariectomized groups $56.6 \pm 10.4$, control group $63.2 \pm 8.2 \mathrm{pg} / \mathrm{l})$, but it did not reached statistical significance $(\mathrm{p}=0.09)$.

Effect of ovariectomy on serum lipids and the effect of treatment with simvastatin are shown in Table 1. Significantly higher serum cholesterol was found in all ovariectomized animals compared to controls. The increase in serum triglycerides was not significant, but we observed the increase in LDL triglyceride content; the ratio triglyceride:cholesterol in LDL particles changed from 1:51.5 in controls to 1:6.5-11.4 in both untreated and treated animals (Table 1).

Simvastatin increased serum level of HDL cholesterol in both treated groups, and especially improved the ratio of total to HDL cholesterol and AIP. However, otherwise it did not substantially change individual serum lipids including LDL cholesterol, serum triglycerides, LDL triglycerides and other serum lipids.

The effect of ovariectomy and treatment with simvastatin on the prevalence of atherosclerotic plaques in abdominal and thoracic aortas is shown in Table 2. Plaques in abdominal and thoracic aortas were found in almost all ovariectomized and untreated animals. In both groups treated with simvastatin lower prevalence of atherosclerotic plaques was observed compared to ovariectomized/non-treated group. In animals treated with simvastatin for 12 weeks the prevalence of atherosclerotic plaques was lower than in ovariectomized animals, but this difference did not reach statistical significance. On the other hand, in animals treated with simvastatin 4 weeks after ovariectomy, the prevalence of atherosclerotic plaques was significantly lower than in untreated animals. 
Table 1. Effects of ovariectomy and statin treatment on serum lipids in golden Syrian hamsters.

\begin{tabular}{lcccc}
\hline Controls & $\mathbf{n}=\mathbf{5}$ & $\begin{array}{c}\text { Ovariectomized } \\
\text { without } \\
\text { treatment } \\
\mathbf{n}=\mathbf{9}\end{array}$ & $\begin{array}{c}\text { Ovariectomized } \\
\text { treated for } \\
\mathbf{1 2} \text { weeks } \\
\mathbf{n = 1 0}\end{array}$ & $\begin{array}{c}\text { Ovariectomized } \\
\text { treated for } \\
\mathbf{8} \text { weeks } \\
\mathbf{n}=\mathbf{9}\end{array}$ \\
\hline Total cholesterol & $4.46 \pm 0.46$ & $5.46 \pm 0.87^{*}$ & $6.05 \pm 1.33^{*}$ & $5.85 \pm 1.80^{*}$ \\
HDL cholesterol & 2.08 & 2.26 & 3.13 & 2.86 \\
LDL cholesterol & 1.03 & 1.33 & 1.77 & 1.72 \\
IDL cholesterol & 0.17 & 0.22 & 0.24 & 0.22 \\
VLDL cholesterol & 0.6 & 0.98 & 0.71 & 0.62 \\
Triglycerides & $1.85 \pm 0.35$ & $2.34 \pm 0.86$ & $1.89 \pm 0.48$ & $1.97 \pm 1.02$ \\
HDL triglycerides & 0.30 & 0.35 & 0.38 & 0.32 \\
LDL triglycerides & 0.02 & 0.18 & 0.27 & 0.15 \\
IDL triglycerides & 0.18 & 0.16 & 0.19 & 0.12 \\
VLDL triglycerides & 1.20 & 1.63 & 1.35 & 1.00 \\
Total/HDL cholesterol ratio & 2.14 & 2.42 & 2.08 & 2.05 \\
AIP** & -0.05 & 0.015 & -0.21 & -0.16 \\
\hline
\end{tabular}

Data are presented in $\mathrm{mmol} / \mathrm{l}$, mean $\pm \mathrm{SD}$. Particular lipid fractions were measured from the pooled blood samples. $* \mathrm{p}<0.05$ vs. Controls; ${ }^{* *}$ Atherogenic index of plasma: $\log ($ triglycerides/HDL)

Table 2. Effects of statin treatment on the presence of atherosclerotic plaques in thoracic and abdominal aorta in golden Syrian hamsters.

\begin{tabular}{|c|c|c|c|c|}
\hline & $\begin{array}{c}\text { Controls } \\
\qquad \begin{array}{l}n=5\end{array}\end{array}$ & $\begin{array}{c}\begin{array}{c}\text { Ovariectomized } \\
\text { without treatment }\end{array} \\
\qquad=9\end{array}$ & $\begin{array}{l}\text { Ovariectomized } \\
\text { treated for } \\
\begin{array}{c}12 \text { weeks } \\
n=10\end{array}\end{array}$ & $\begin{array}{c}\text { Ovariectomized } \\
\text { treated for } \\
8 \text { weeks } \\
\text { n=9 }\end{array}$ \\
\hline $\begin{array}{l}\text { Animals with } \\
\text { plaques }\end{array}$ & 0 & $7(78 \%)^{*}$ & $5(50 \%)$ & $2(22 \%) \dagger$ \\
\hline
\end{tabular}

* $p<0.01$ Ovariectomized without treatment vs. Controls; $+p<0.05$ Ovariectomized treated for 8 weeks vs. Ovariectomized without treatment

\section{Discussion}

The surgical menopause (ovariectomy) significantly increased total cholesterol and the corresponding increase in serum LDL cholesterol was observed in pooled samples. The most substantial increase was observed in the content of triglycerides in LDL fraction. However, the content of triglycerides in LDL changed only in order of several percents and we cannot exclude, that at least partly it could be caused by variability of measurements in pooled samples. Nevertheless, it should be noted that this increase was detected in all three ovariectomized groups.

Simvastatin increased mainly HDL cholesterol, but it did not substantially affect the content of triglycerides in LDL particles. The effect of simvastatin was even more prominent on total/HDL cholesterol ratio and AIP, thus underscoring the importance of additional lipid parameters to LDL cholesterol, especially in females.

Surgical menopause increased the prevalence of atherosclerotic changes in aortas of golden Syrian hamster in a very short period. These changes were not completely abolished by simvastatin and the time of initiation of simvastatin treatment after ovariectomy had no significant impact on the changes in lipoproteins, whilst it led to lower prevalence of atherosclerotic changes. The atherosclerotic changes were less prevalent 
in animals treated for shorter period and with delay. However, this difference did not reach statistical significance.

Our study confirmed already described increase in total cholesterol and increase in atherosclerotic changes caused by ovariectomy in golden Syrian hamster (Nistor et al. 1987, Sohn et al. 1999). In contrast to previous study (El-Swefy et al. 2000), in which treatment with lovastatin led to decrease of LDL cholesterol, in our study simvastatin was not sufficient to prevent ovarian hormone deficiency-induced hypercholesterolemia. The explanation is that the effect of ovariectomy on the statin treatment was not studied in previous projects. Therefore, we can hypothesize that in this case the statin treatment prevented atherosclerotic changes rather through an increase in HDL cholesterol. Another possibility of different results is the change in the composition of LDL particles caused by ovariectomy.

The finding, that decrease in atherosclerotic changes was not accompanied by decrease of LDL cholesterol, was rather surprising and could be speciesspecific. However, even in human studies, LDL cholesterol in women was already described as less predictive of subsequent cardiovascular events, while triglycerides and HDL cholesterol were stronger predictors (Bass et al. 1993).

The strength of our study is the analysis of the impact of lipoprotein changes in menopause on the efficacy of statins under standardized laboratory conditions in a well established experimental model and by the timing of exploration of atherosclerotic and metabolic changes just after the menopause, the period probably most critical for atherosclerotic changes.

The limitation of our study is the use of experimental model, which always has certain limitations. The lack of effect on LDL cholesterol could be species-specific, however, from the point of view of lipid disorders this experimental model was already proved to be very close to human metabolism. In addition, pooled serum samples for analyses of lipid subfractions allowed us only approximate assessment of the real significance of particular lipoprotein subfractions. We were also not able to offer more detailed view of lipid changes during experimentally induced menopause. For example, lipoprotein fractions and cholesterol esters were not measured individually, which would give a better insight into cholesterol ester transfer protein activity.

In conclusion, simvastatin treatment in this experimental study prevented atherosclerotic changes induced by ovariectomy; however, this effect was not associated with decrease in LDL cholesterol. This finding might indicate, that serum LDL cholesterol could not be always the only lipid target for successful prevention of cardiovascular diseases in perimenopause and studies in women should be more focused also on proportions of plasma/serum lipids expressed as total cholesterol/HDL cholesterol or AIP.

\section{Conflict of Interest}

There is no conflict of interest.

\section{Acknowledgements}

The work was supported by institutional finance support from the Institute for Clinical and Experimental Medicine (MZO 00023001) and by research grant 1M0510 (Ministry of Education, Youth and Sports of the Czech Republic).

\section{References}

ARENAS IA, ARMSTRONG SJ, XU Y, DAVIDGE ST: Tumor necrosis factor-alpha and vascular angiotensin II in estrogen-deficient rats. Hypertension 48: 497-503, 2006.

BASS KM, NEWSCHAFFER CJ, KLAG MJ, BUSH TL: Plasma lipoprotein levels as predictors of cardiovascular death in women. Arch Intern Med 153: 2209-2216, 1993.

CLARKSON TB: Estrogen effects on arteries vary with stage of reproductive life and extent of subclinical atherosclerosis progression. Menopause 14: 373-384, 2007.

DOBIÁŠOVÁ M, FROHLICH J: The plasma parameter log (TG/HDL-C) as an atherogenic index: correlation with lipoprotein particle size and esterification rate in apoB-lipoprotein-depleted plasma (FER(HDL)). Clin Biochem. 34: 583-588, 2001.

EL-SWEFY S, SCHAEFER EJ, SEMAN LJ, VAN DONGEN D, SEVANIAN A, SMITH DE, ORDOVAS JM, ELSWEIDY M, MEYDANI M: The effect of vitamin E, probucol, and lovastatin on oxidative status and aortic fatty lesions in hyperlipidemic-diabetic hamsters. Atherosclerosis 149: 277-286, 2000. 
FROHLICH J, DOBIÁŠOVÁ M, ADLER L, FRANCIS M: Gender differences in plasma levels of lipoprotein (a) in patients with angiographically proven coronary artery disease. Physiol Res 53: 481-486, 2004.

HAVEL RJ, EDER HA, BRAGDON JH: The distribution and chemical composition of ultracentrifugally separated lipoproteins in human serum. J Clin Invest 34: 1345-1353, 1955.

KOVÁŘ J, POLEDNE R: Sex differences in the response of postprandial lipemia to a change from a low-fat lowcholesterol diet to a high-fat high-cholesterol diet. Physiol Res 49: 233-239, 2000.

LUCAS EA, KHALIL DA, DAGGY BP, ARJMANDI BH: Ethanol-extracted soy protein isolate does not modulate serum cholesterol in golden Syrian hamsters: a model of postmenopausal hypercholesterolemia. $J$ Nutr 131: 211-214, 2001.

MILCENT C, DORMONT B, DURAND-ZALESKI I, STEG PG: Gender differences in hospital mortality and use of percutaneous coronary intervention in acute myocardial infarction: microsimulation analysis of the 1999 nationwide French hospitals database. Circulation 115: 833-839. 2007.

NISTOR A, BULLA A, FILIP DA, RADU A: The hyperlipidemic hamster as a model of experimental atherosclerosis. Atherosclerosis 68: 159-173, 1987.

RAZA JA, REINHART RA, MOVAHED A: Ischemic heart disease in women and the role of hormone therapy. Int $J$ Cardiol 96: 7-19, 2004.

SOHN E, DAGGY BP, ARJMANDI BH: Ovariectomized hamster: a potential model of postmenopausal hypercholesterolemia. J Nutr Biochem 10: 660-663, 1999.

VRÁBLÍK M, FAIT T, KOVÁŘ J, POLEDNE R, ČEŠKA R: Oral but not transdermal estrogen replacement therapy changes the composition of plasma lipoproteins. Metabolism 57: 1088-1092, 2008.

WALSH JM, PIGNONE M: Drug treatment of hyperlipidemia in women. JAMA 29: 2243-2252, 2004.

WILSON TA, NICOLOSI RJ, LAWTON CW, BABIAK J: Gender differences in response to a hypercholesterolemic diet in hamsters: effects on plasma lipoprotein cholesterol concentrations and early aortic atherosclerosis. Atherosclerosis 146: 83-91, 1999. 